\title{
Fourier Based Adaptive Waveform
}

\author{
Marwa Chafii *, Jacques Palicot *, Rémi Gribonval ${ }^{\dagger}$, and Faouzi Bader * \\ * CentraleSupélec, IETR, Campus de Rennes \\ 35576 Cesson - Sévigné Cedex, France \\ Email: \{marwa.chafii, jacques.palicot, faouzi.bader\} @ centralesupelec.fr \\ †Univ Rennes, Inria, CNRS, IRISA \\ 35042 Rennes Cedex, France \\ Email: remi.gribonval@inria.fr
}

\begin{abstract}
The single carrier-frequency domain equalization (SC-FDE) scheme and the orthogonal frequency division multiplexing (OFDM) system have complementary performance benefits that make it hard to choose one or the other. This paper proposes an alternative waveform called Fourier-based adaptive waveform (FAW), which adapts the time-frequency tiling of the modulated signal to the frequency fading properties of the environment. A performance comparison based on peak-to-average power ratio and bit error rate shows that the proposed scheme achieves a good trade-off between SC-FDE and OFDM.

Index Terms-Adaptive waveforms, Fourier Transform, OFDM, SC-FDE, DFT-Precoding, Peak-to-Average Power ratio (PAPR)
\end{abstract}

\section{INTRODUCTION AND MOTIVATIONS}

The discussion on the single carrier and the multi-carrier waveforms dates back to the last decades [1]-[3], where several researchers have been supporting the single-carrier scheme, and others seeing more advantages in the multi-carrier schemes. On one hand, the choice of the single carrier-frequency domain equalization (SC-FDE) system has been fostered thanks to its reduced peak-to-average power ratio (PAPR) requirements with respect to the classical multi-carrier system namely orthogonal frequency division multiplexing (OFDM), thereby allowing the use of more power-efficient amplifiers. On the other hand, the study on OFDM, as well as its adoption by numerous wireless and wireline communication standards, has been motivated by its resilience against the hostility of frequency selective fading channels, and its low complexity channel equalization. An arrangement of operating a dual-mode system has been made in long term evolution (LTE) standard as reported in 3GPP Releases 8/9 [4], wherein the base station employs an OFDM transmitter and an SC-FDE receiver, and the user modem employs an SC-FDE transmitter and an OFDM receiver. The advantage of this SC-FDE uplink and OFDM downlink mode, is that the subscriber power consumption is low, and most of signal processing complexity is offloaded to the base station.

In this paper, we propose a new adaptive waveform named Fourier-based adaptive waveform (FAW) as a solution of the single-carrier/multi-carrier trade-off, which achieves an intermediate system performance between these two conventional waveforms. Section II defines and explains the principle of FAW transceiver, the time-frequency tiling properties as well as the analytical expression of the resulting modulated signal. Section III provides the PAPR and the bit error rate (BER) per- formance in frequency selective channel for the new proposed waveform, and compares them with coded OFDM and coded SC-FDE. Section IV concludes the paper and suggests future directions of this work.

\section{FAW PRINCIPLE}

\section{A. Overview}

Let $\mathcal{B}$ be the total allocated spectral bandwidth. We assume that $\mathcal{B}$ can be divided into $M$ sub-bands that we name elementary frequencies $f_{m}$ :

$$
\mathcal{B}=\bigcup_{m=0}^{M-1} f_{m} .
$$

OFDM divides the bandwidth $\mathcal{B}$ into $M$ elementary frequencies also known as $M$ sub-carriers. If the channel suffers from a deep frequency fading around some frequencies, it will not impact the other frequencies since all the sub-carriers are well frequency localized. Therefore, the OFDM provides robustness against frequency selective fading channels. Nevertheless, the addition of multiple sub-carriers in time generates envelope fluctuations, which cause large PAPR. Unlike OFDM, SC-FDE does not divide the bandwidth, since each data symbol occupies fully the bandwidth, which make all the symbols vulnerable to channel frequency fading. Furthermore, at each instant, only one symbol is transmitted over time, and hence generating a lower PAPR. By making the observation that the channel deep fading is usually restricted around few parts of the bandwidth, we can think about isolating only these damaged frequencies from the rest of the spectrum instead of isolating each single frequency from the others as implemented in OFDM. By acting this way, we prevent a large PAPR. Based on a channel state information (CSI) at the transmitter side, the FAW waveform proposed in this study divides the bandwidth into elementary frequencies around the attenuated frequencies by the channel, and keeps the rest of the bandwidth undivided. The FAW waveform can be seen as a mixture or a hybrid design of the single-carrier and the multi-carrier. It allows the use of a multi-carrier structure around the frequencies affected by the channel fading, and a single-carrier structure around the non-affected frequencies. Acting this way, the general PAPR performance is improved compared with OFDM, and the BER 


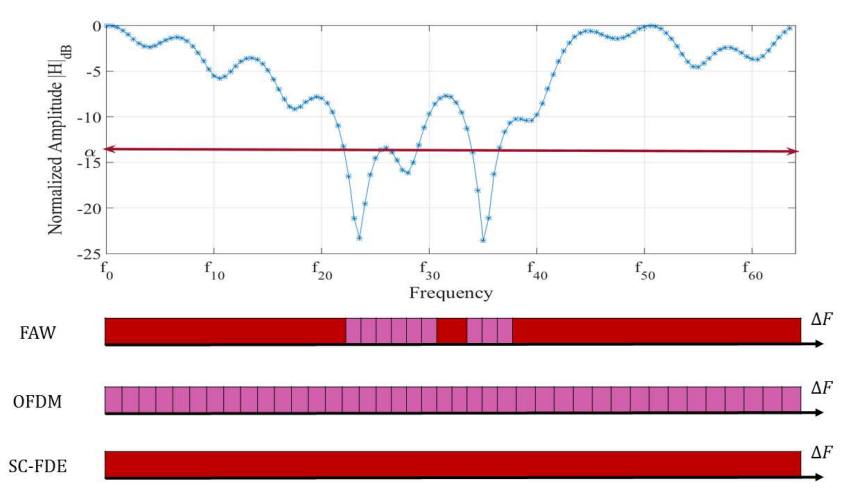

Figure 1: FAW bandwidth repartition

OFDM

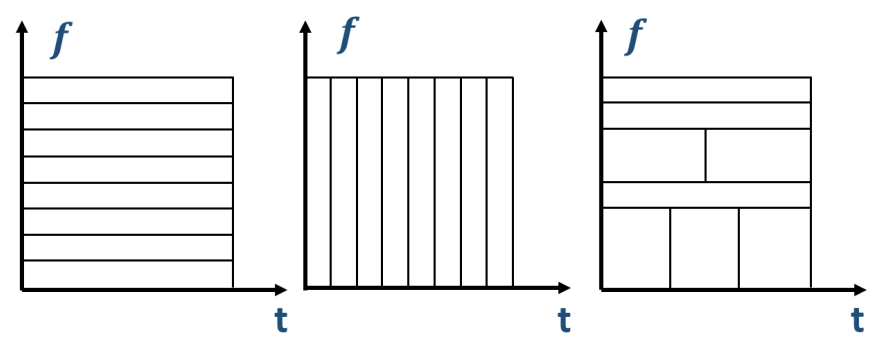

Figure 2: Example of flexible time-frequency tiling of FAW

performance is enhanced with respect to SC-FDE as shown further in Section III.

The first step of creating the FAW modulated signal, is to label the elementary frequencies as "attenuated" or "nonattenuated". One possible approach is to compare the normalized amplitude of the frequency channel response $\left|H\left(f_{m}\right)\right|$ of the associated frequency $f_{m}$ with a pre-defined threshold $\alpha_{\mathrm{dB}} \in \mathbb{R}$. Then, we state that the elementary frequency $f_{m}$ is "non-attenuated" by the channel if $\left|H\left(f_{m}\right)\right|_{\mathrm{dB}} \geq \alpha$, otherwise, it is stated "attenuated". The next step is to construct a Fourier based signal that provides a good frequency localization around the attenuated frequencies. A schematic representation of the resulting bandwidth of FAW scheme for a frequency selective fading channel is depicted in Fig. 1. The well-known OFDM and the SC-FDE bandwidth repartition are also provided for comparison. It is worth noticing that when $\alpha_{\mathrm{dB}}=0 \mathrm{~dB}$, FAW becomes OFDM, and when $\alpha_{\mathrm{dB}}$ is set to $-\infty \mathrm{dB}$, FAW becomes equivalent to SC-FDE.

\section{B. Time-frequency tiling}

In order to provide an insight into the time-frequency properties of the studied schemes, Fig. 2 depicts the time-frequency boxes, also known as "Heisenberg rectangles" [5], which reflects the time localization and the frequency localization of OFDM-based, SC-FDE-based, and FAW modulated signals. Note that each box carries one input data symbol. While OFDM
(SC-FDE respectively) provides a regular time-frequency grid, where each box has narrow bandwidth and long time duration (wide bandwidth and short time duration respectively), FAW is able to offer a very flexible time-frequency tiling, controlled only by the time-frequency trade-off expressed by the uncertainty principle [6].

\section{FAW Transceiver}

In order to achieve a flexible time-frequency tiling as provided in Fig. 2, FAW signal can be implemented efficiently using a discrete Fourier transform (DFT) based precoding along with an inverse discrete Fourier transform (IDFT) processing. The FAW modulation can then be represented as an adaptive precoded OFDM as shown in Fig. 3. The precoding block takes as input channel state information (CSI) and the attenuation threshold $\alpha$. Based on CSI, the elementary frequencies are labelled as attenuated or non-attenuated with respect to the value of $\alpha$. It is worth mentioning that some variants of DFTbased precoded OFDM have been proposed in the literature for different purposes such as coexistence scenarios in professional mobile radio services [7] and single-carrier frequency division multiple access [8].

The precoding applies a global DFT to the adjacent nonattenuated elementary frequencies as described in Fig. 4. For a number of carriers equal to $M=8$, and a predefined $\alpha$, we assume that $\left\{f_{0}, f_{1}, f_{2}, f_{4}, f_{5}\right\}$ are non-attenuated frequencies, while the rest of the frequencies are affected by the channel fading, based on the current CSI available at the receiver. As depicted in Fig. 4, a 3-point DFT (2-point DFT respectively) is applied to the input symbol vector $\left[x_{0}, x_{1}, x_{2}\right]$ (to $\left[x_{4}, x_{5}\right]$ respectively). A global IDFT is then applied to the precoded symbol vector. The resulting bandwidth repartition shows that the attenuated frequencies are well frequency-localized and thus isolated from the rest of the frequencies.

\section{FAW signal expression}

Let $x=\left\{x_{j}\right\}_{j=0}^{M-1} \in \mathbb{C}^{M}$ be the input data symbols vector of length $M$. We assume that the final repartition of the bandwidth is a partition of $B$ sub-bands, each of length $\left\{L_{b}\right\}_{b=0}^{B-1}$. Then we have $\sum_{b=0}^{B-1} L_{b}=M$. The input vector $x$ can be represented as:

$$
x=\left(\begin{array}{c}
x^{0} \\
x^{1} \\
\vdots \\
x^{b} \\
\vdots \\
x^{B-1}
\end{array}\right),
$$

such that

$$
x^{b} \in \mathbb{C}^{L_{b}}=\left(x_{m}^{b}\right)_{m=0}^{L_{b}-1} .
$$




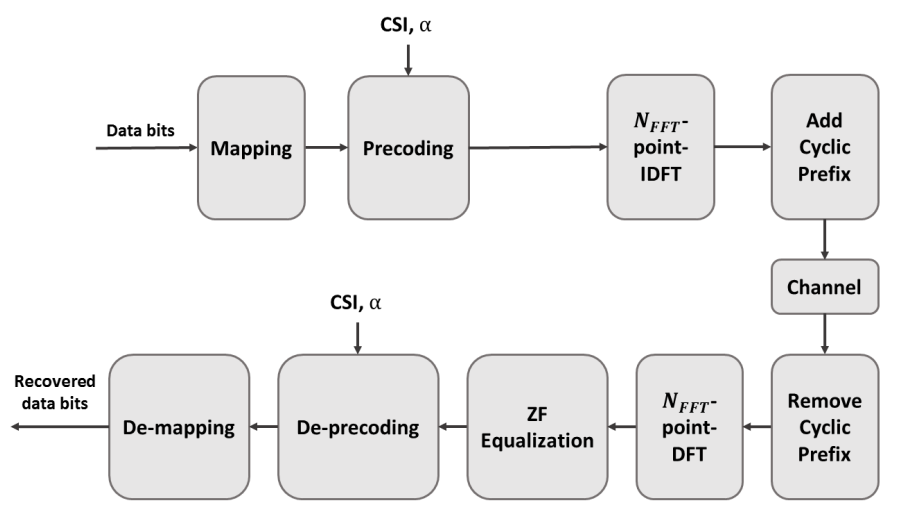

Figure 3: FAW transceiver

Let $u^{b}:=\operatorname{DFT}\left(x^{b}\right)$ be the output of the DFT block for a subband $b$. The total output vector of the DFT-precoding is then:

$$
u=\left(\begin{array}{c}
u^{0} \\
\vdots \\
u^{b} \\
\vdots \\
u^{B-1}
\end{array}\right)
$$

Note that if the elementary frequency associated to a sub-band $b$ is not attenuated, we have $L_{b}=1$, and thus $u^{b}:=x^{b}$.

Let $X(n)$ be the FAW transmitted signal, then we have

$$
\begin{aligned}
X(n) & =\operatorname{IDFT}(u) \\
& =\frac{1}{M} \sum_{k=0}^{M-1} u_{k} \exp \left(2 i \pi \frac{k n}{M}\right) \\
& =\frac{1}{M} \sum_{k=0}^{M-1} u_{l(k)}^{b(k)} \exp \left(2 i \pi \frac{k n}{M}\right),
\end{aligned}
$$

where $l(k) \in \llbracket 0, L_{b(k)}-1 \rrbracket$. Moreover

$$
\begin{aligned}
& u_{l(k)}^{b(k)}=\sum_{m=0}^{L_{b(k)}-1} x_{m}^{b(k)} \exp \left(-2 i \pi \frac{m l(k)}{L_{b(k)}}\right), \\
& \text { where } \quad x_{m}^{b(k)}=x_{j}, \\
& \text { such that } \quad j=m+\sum_{b=0}^{b(j)-1} L_{b} .
\end{aligned}
$$

Finally,

$$
X(n)=\frac{1}{M} \sum_{k=0}^{M-1} \sum_{m=0}^{L_{b(k)}-1} x_{m}^{b(k)} \exp \left(2 i \pi\left(\frac{k n}{M}-\frac{m l(k)}{L_{b(k)}}\right)\right) .
$$

As an example, we consider the FAW scheme $(M=8)$ in Fig. 4. The parameters that allow to express the FAW transmitted signal are provided in Table I.

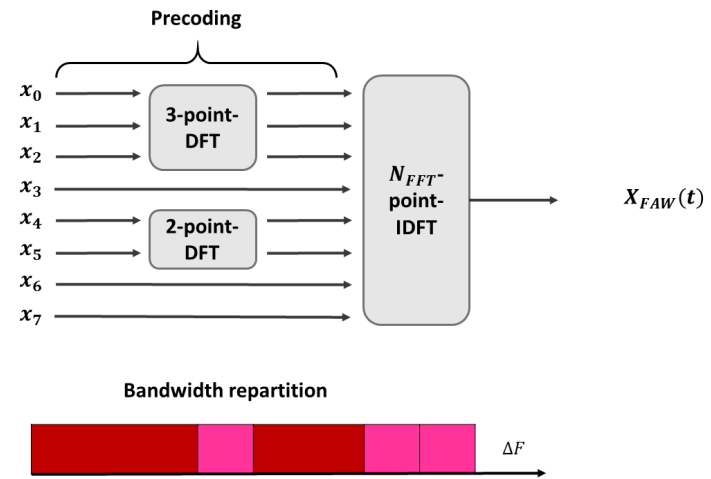

Figure 4: FAW precoding operation

Table I: Example of the parameters values for FAW signal

\begin{tabular}{|c|c|c|c|}
\hline$k$ & $L_{b(k)}$ & $l(k)$ & $b(k)$ \\
\hline 0 & 3 & 0 & 0 \\
\hline 1 & 3 & 1 & 0 \\
\hline 2 & 3 & 2 & 0 \\
\hline 3 & 1 & 0 & 1 \\
\hline 4 & 2 & 0 & 2 \\
\hline 5 & 2 & 1 & 2 \\
\hline 6 & 1 & 0 & 3 \\
\hline 7 & 1 & 0 & 4 \\
\hline
\end{tabular}

Table II: Simulation parameters

\begin{tabular}{|c|c|c|}
\hline Parameter & Definition & Value \\
\hline$M$ & $\begin{array}{c}\text { Number of the elementary } \\
\text { frequencies (sub-carriers) }\end{array}$ & 64 \\
\hline$\Delta F$ & Elementary frequency spacing & $15 \mathrm{kHz}$ \\
\hline $\mathcal{M}$ & $\mathcal{M}$-QAM Constellation & 4 \\
\hline $\mathrm{CP}$ & Length of cyclic prefix & 16 \\
\hline$r$ & $\begin{array}{c}\text { Roll-off of the root raised cosine } \\
\text { filter }\end{array}$ & 0.3 \\
\hline$L$ & Oversampling factor & 4 \\
\hline$\nu$ & $\begin{array}{c}\text { Number of paths of the Rayleigh } \\
\text { fading channel }\end{array}$ & $4-\infty,-20,0\}$ \\
\hline$\alpha_{\mathrm{dB}}$ & Threshold of attenuation in dB \\
\hline$N_{\text {frames }}$ & $\begin{array}{c}\text { Number of frames (each of } M \\
\text { symbols) }\end{array}$ & $10^{6}$ \\
\hline
\end{tabular}

\section{Simulation RESULTS}

In this section, the PAPR and the BER performance are evaluated using the parameters reported in Table II.

The signals of the compared schemes are sent through a frequency selective Rayleigh fading channel represented by the channel impulse response coefficients: $h=(h(1), h(2) \ldots h(\nu))$, such that $\nu$ is the number of paths. We assume that all the coefficients are circularly symmetric complex Gaussian random variables with $\mathcal{C N}\left(0, \frac{1}{\nu}\right)$ distribution.

\section{A. PAPR performance}

The PAPR performance is evaluated in Fig. 5, through the complementary cumulative distribution function (CCDF) which 


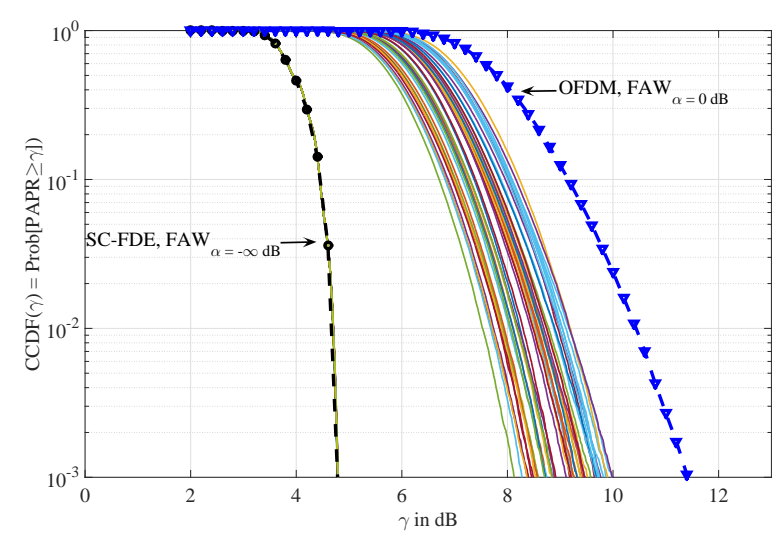

Figure 5: PAPR performance comparison for SC-FDE, OFDM, and $\mathrm{FAW}_{\alpha=-20 \mathrm{~dB}}$ for different channel realizations.

is the probability that the PAPR exceeds a certain value $\gamma$. A hundred channel realizations of the Rayleigh fading channel $h$ have been evaluated for $\mathrm{FAW}_{\alpha=-20 \mathrm{~dB}}$ and their corresponding CCDF are plotted in straight lines in Fig. 5. SC-FDE and OFDM are also depicted in the same figure as benchmarks. For each realization of the channel, the frequencies are classified as attenuated and non-attenuated, and then a DFT-based precoding is applied followed by an IDFT. Each realization provides different channel characteristics, thereby leading to different bandwidth repartitions. Therefore, different PAPR performance are produced as can be witnessed in the figure. Depending on the channel characteristics, FAW FW $_{\alpha=-20 \mathrm{~dB}}$ exhibits a PAPR performance gain (for $\mathrm{CCDF}=10^{-3}$ ) varying from $1.5 \mathrm{~dB}$ to $7 \mathrm{~dB}$ compared with OFDM. The main reason of this advantage is that, at each instant, the number of sub-carriers overlapping in time is usually less than in OFDM case. With reference to SC-FDE, the PAPR performance loss fluctuates between 0 and $5.2 \mathrm{~dB}$ depending on how deep is the frequency fading of the channel response compared with the threshold $\alpha_{\mathrm{dB}}$. In fact, the channel realization that reaches the same PAPR performance than SC-FDE, satisfies : $\forall f_{m} \in \mathcal{B}\left|H\left(f_{m}\right)\right| \geq \alpha$, therefore, all the frequencies are labelled as non-attenuated, and there is no need to divide the bandwidth which leads to the SC-FDE case.

Note that there is no CCDF curve resulting from any channel realization, between $\gamma_{1}=4.8 \mathrm{~dB}$ and $\gamma_{2}=8.1 \mathrm{~dB}$. In fact, $\gamma_{1}$ results from a non-divided bandwidth, and $\gamma_{2}$ is achieved when there is an occurrence of at least one attenuated elementary frequency. This fact supports the high sensitivity of the PAPR performance with respect to the number of overlapping subcarriers in time.

\section{B. BER performance}

The BER performance of the same 100 channel realizations studied in Section III-A has been evaluated for the FAW $_{\alpha=-20 \mathrm{~dB}}$ scheme in Fig. 6. A convolutional coding of $\frac{1}{2}$ code rate, along with a Viterbi detection have been employed in order to provide a more realistic assessment. As it can be

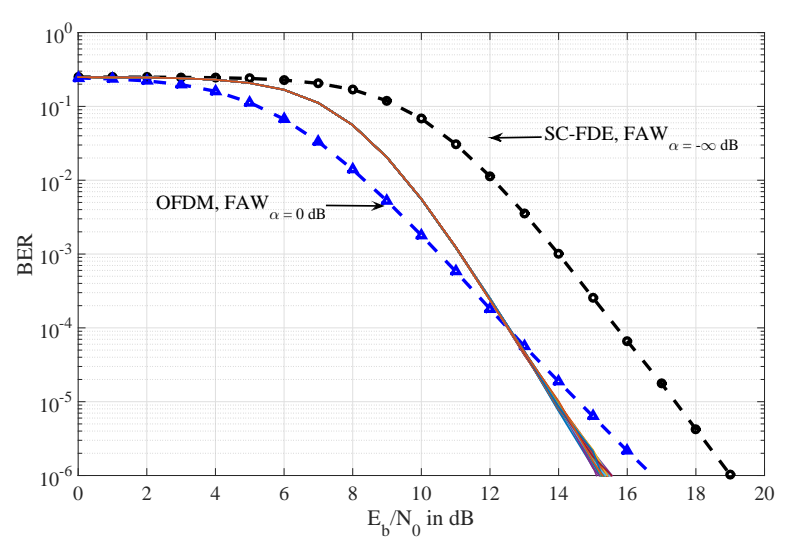

Figure 6: BER performance comparison for SC-FDE, OFDM, and $\mathrm{FAW}_{\alpha=-20 \mathrm{~dB}}$ for different channel realizations.

observed from Fig. 6, the FAW ${ }_{\alpha=-20 \mathrm{~dB}}$ curves for the different channel realizations overlap. This can be intuitively explained by the consideration that fixing the same threshold $\alpha$ for the different channels, and well-localizing the frequencies in deep fading, give the overall system more or less the same fading level and hence the same BER performance. Moreover, we notice that:

- For a normalized signal-to-noise ratio $E_{b} / N_{0} \leq 12.5 \mathrm{~dB}$, the BER performance of $\mathrm{FAW}_{\alpha=-20 \mathrm{~dB}}$ are significantly improved with respect to SC-FDE ( $3 \mathrm{~dB}$ of $E_{b} / N_{0}$ gain for $\left.\mathrm{BER}=10^{-3}\right)$, whereas OFDM clearly outperforms FAW $_{\alpha=-20 \mathrm{~dB}}\left(0.5 \mathrm{~dB}\right.$ of $E_{b} / N_{0}$ gain for BER $\left.=10^{-3}\right)$.

- For $E_{b} / N_{0}>12.5$, the BER performance of FAW $\alpha=-20 \mathrm{~dB}$ surprisingly exceeds both OFDM and SC-FDE. This result encourages further investigations to characterize this second regime.

\section{CONCLUSION}

This paper proposes a new adaptive waveform as an alternative to OFDM and SC-FDE schemes. The principle of FAW consists in performing an adaptive DFT-based precoding depending on channel conditions, which provides a flexible time-frequency tiling of the resulting signal. Based on this flexibility, the FAW schemes include SC-FDE, OFDM, and several schemes between these extreme cases. For a predefined threshold of attenuation, and an available channel state information, FAW employs the scheme that leads automatically to the best performance trade-off. The key performance in this paper includes PAPR and BER performance.

Our future work is to dynamically identify the threshold of attenuation in order to improve the bandwidth partitioning for a given CSI and a performance trade-off. Another perspective of the work can exploit fully the CSI by using other adaptive techniques such as rate adaptation and power allocation over sub-bands. 


\section{ACKNOWLEDGMENT}

This work has received a French state support granted to the Enhanced PHY for cellular Low power communication IoT (EPHYL) project and managed by the National Research Agency under reference Nb. ANR-16-CE25-0002-03.

\section{REFERENCES}

[1] Andreas Czylwik. Comparison between adaptive OFDM and single carrier modulation with frequency domain equalization. In Vehicular Technology Conference, 1997, IEEE 47th, volume 2, pages 865-869. IEEE, 1997.

[2] Zhengdao Wang, Xiaoli Ma, and Georgios B Giannakis. OFDM or singlecarrier block transmissions? IEEE Transactions on Communications, 52(3):380-394, 2004.

[3] Lei Ye and Alister Burr. Frequency diversity comparison of coded SC-FDE \& OFDM on different channels. In Personal, Indoor and Mobile Radio Communications, 2007. PIMRC 2007. IEEE 18th International Symposium on, pages 1-5. IEEE, 2007.

[4] Magdalena Nohrborg. LTE Overview. 3GPP, A Global Initiative, THE Mobile Broadband Standard, August 2013.

[5] Stéphane Mallat. A Wavelet Tour of Signal Processing. Academic press, 2008.

[6] NG De Bruijn. Uncertainty Principles in Fourier Analysis. Inequalities, 2:57-71, 1967.

[7] Markku Renfors, Faouzi Bader, Leonardo Baltar, Didier Le Ruyet, Daniel Roviras, Philippe Mege, Martin Haardt, and Tobias Hidalgo Stitz. On the use of filter bank based multicarrier modulation for professional mobile radio. In Vehicular Technology Conference (VTC Spring), 2013 IEEE 77th, pages 1-5. IEEE, 2013.

[8] Ian C Wong, Oghenekome Oteri, and Wes McCoy. Optimal resource allocation in uplink SC-FDMA systems. IEEE Transactions on Wireless communications, 8(5), 2009. 